\title{
A Dynamic Phase-field Model for Structural Transformations and Twinning: Regularized Interfaces with Transparent Prescription of Complex Kinetics and Nucleation. Part II: Two-Dimensional Characterization and Boundary Kinetics.
}

\author{
Vaibhav Agrawal* and Kaushik Dayal ${ }^{\dagger}$ \\ Carnegie Mellon University
}

March 27, 2015

\begin{abstract}
A companion paper presented the formulation of a phase-field model - i.e., a model with regularized interfaces that do not require explicit numerical tracking - that allows for easy and transparent prescription of complex interface kinetics and nucleation. The key ingredients there were a re-parametrization of the energy density to clearly separate nucleation from kinetics; and an evolution law that comes from a conservation statement for interfaces. This enables clear prescription of nucleation through the source term of the conservation law and of kinetics through an interfacial velocity field. This model overcomes an important shortcoming of existing phase-field models, namely that the specification of kinetics and nucleation is both restrictive and extremely opaque.

In this paper, we present a number of numerical calculations - in one and two dimensions - that characterize our formulation. These calculations illustrate: (i) highly-sensitive rate-dependent nucleation; (ii) independent prescription of the forward and backward nucleation stresses without changing the energy landscape; (iii) stick-slip interface kinetics (iii) the competition between nucleation and kinetics in determining the final microstructural state; (iv) the effect of anisotropic kinetics; and (v) the effect of non-monotone kinetics. These calculations demonstrate the ability of this formulation to precisely prescribe complex nucleation and kinetics in a simple and transparent manner.

We also extend our conservation statement to describe the kinetics of the junction lines between microstructural interfaces and boundaries. This enables us to prescribe an additional kinetic relation for the boundary, and we examine the interplay between the bulk kinetics and junction kinetics.

Keywords: Phase-field modeling, Twinning, Structural phase transformation, Nucleation of Interfaces, Kinetics of Interfaces
\end{abstract}

*vaibhava@andrew. cmu.edu

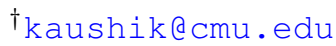




\section{Introduction}

In the companion paper, we presented the formulation and one-dimensional characterization of a phasefield model focused on structural transformations and twinning. The key new feature of our model is the ability to transparently and easily prescribe complex nucleation and kinetic behavior of microstructural interfaces. While the one-dimensional setting provides a simplified arena to transparently understand and characterize certain aspects, many essential features of structural transformations and twinning are inherently higher-dimensional. In particular, elastic compatibility is trivial in one dimension, but can be central to the behavior of interfaces in 2 and 3 dimension [BJ87, BJ92, Bha03].

The key issue can be illustrated in a simple example. Consider a one-dimensional setting with a material that has two stress-free strains $\varepsilon_{1}$ and $\varepsilon_{2}$. Given traction-free boundary conditions, any spatial arrangement of $\varepsilon_{1}$ and $\varepsilon_{2}$ will satisfy both equilibrium as well as elastic compatibility. On the other hand, consider a $2 \mathrm{D}$ or $3 \mathrm{D}$ system with phases given by stress-free stretches $\boldsymbol{U}_{1}$ and $\boldsymbol{U}_{2}$, and with traction-free boundaries. If these do not satisfy the kinematic compatibility condition - i.e. there is no solution $\boldsymbol{Q}, \boldsymbol{a}, \boldsymbol{n}$ to the equation $\boldsymbol{Q} \boldsymbol{U}_{1}-\boldsymbol{U}_{2}=\boldsymbol{a} \otimes \boldsymbol{n}$ - then the only stress-free solutions are either uniformly $\boldsymbol{U}_{1}$ or uniformly $\boldsymbol{U}_{2}$ in the entire domain. Any solution with both phases will involve stresses or elastodynamics, or both.

The focus of this paper is to characterize the model in 2 dimensions where the kinetics and nucleation of interfaces interacts strongly with complex stress fields. These stress fields can arise when phases are not stress-free compatible. They can also emerge during the nucleation process even if the phases are stress-free compatible; from the equation $\boldsymbol{Q} \boldsymbol{U}_{1}-\boldsymbol{U}_{2}=\boldsymbol{a} \otimes \boldsymbol{n}$, we note that only specific rotations and interface directions are stress-free, and the nucleation process is unlikely to follow precisely that loading path. The examples that we consider in this paper include both of these possible effects.

Some of the specific examples, and the motivations for considering them, are as follows.

First, we examine the effect of a non-monotone kinetic relation both in 1D and 2D. Previous work analyzing non-monotone kinetic relations has shown extremely interesting features such as the natural emergence of stick-slip behavior [RK97]. That motivates us to study this example; an important caveat however is that [RK97] use a driving force that is a non-monotone function of interface velocity, whereas we are restricted to setting the interface velocity to be a non-monotone function of the driving force. Possibly for this reason, we do not observe as rich a behavior as [RK97].

Second, we present the formulation of a model that can display anisotropic kinetics; while we use a simple functional form of anisotropy, it is readily generalizable to more complex settings. A key motivation for this example is that twin boundary propagation is extremely anisotropic [AV03].

Third, we present the formulation of a model that displays stick-slip behavior of interfaces, i.e., it requires a finite value of driving force to initiate motion. This is a universal feature of microstructural interfaces, but is challenging to incorporate in standard phase-field models. Recent approaches in higher dimensions use spatially-oscillating imposed stress fields [LL07, LLP10]. In these strategies, it is not clear how - or even if it is possible - to incorporate the anisotropy of the stick-slip threshold that is widely observed. An additional challenge is the requirement of a fine discretization to resolve the imposed stress field.

Fourth, we examine the question of nucleation in 1D and 2D, through the vehicle of computing hysteresis loops. We demonstrate that it is simple and transparent to impose complex nucleation criteria that are ratedependent as well as different for the forward and reverse transformation. This is a particular advantage of our model over existing phase-field approaches; in the latter, nucleation criteria are adjusted in an $a d$ - 
hoc manner by modifying barriers in the energy landscape. Modifying barriers can have consequences on both reverse and forward transformations, is extremely unsystematic, and cannot allow for complex nucleation criteria based on rates and stress measures such as hydrostatic stress.

Finally, we examine the competition between nucleation and kinetics by varying the relative rates of these processes. This provides a powerful tool to systematically examine the effect of different types of microstructure evolution on the final microstructure.

We also extend our model to the kinetics of the junction lines that form at the intersection of microstructural interfaces and material surfaces such as free surfaces. In general, junction lines can be considered as distinct defects with their own kinetics, though coupled to the interface kinetics through the evolution of the stress field. Experimental measurements, many of which are on the surface of a specimen, will therefore largely probe the junction kinetics and not the interface kinetics. Hence, it is important to construct a model that can incorporate a distinct kinetics for junction lines. In analogy to the formulation in the companion paper, we develop a model that allows for the independent prescription of the kinetics of the junction lines, while at the same time treating them as regularized non-singular defects. Our approach builds on previous work by [SB98] that treats interfaces and junctions as singular objects. Our point of departure is to treat these defects in a regularized setting while still allowing for the independent prescription of interface and junction kinetics.

For a list of notation, we refer to the companion paper.

\subsection{Organization}

The paper is organized as follows.

- In Section 2, we briefly recall our proposed model that is described in greater detail in the companion paper.

- In Section 3, we outline the formulation of a 2D energy density that we use in many of the subsequent $2 \mathrm{D}$ calculations.

- In Section 4, we examine the effect of non-monotone kinetic response in 1D and 2D.

- In Section 5, we demonstrate the formulation of anisotropic kinetic laws in 2D.

- In Section 6, we examine twinning interfaces with stick-slip kinetics in 2D.

- In Section 7, we demonstrate - in 1D and 2D - the formulation of complex nucleation criteria that includes rate-dependent critical nucleation stresses, as well as independent prescription of forward and reverse nucleation stresses, without modifying the energy barriers.

- In Section 8, we demonstrate imposing hydrostatic stress-dependence of twin nucleation, in addition to usual shear-stress dependence.

- In Section 9, we examine the competition between nucleation and kinetics in a twinning transformation.

- In Section 10, we examine the prescription of a kinetics associated with the junctions between interfaces and specimen boundaries. 
- In Section 11, we review our work.

\section{Summary of the Proposed Phase-Field Model}

Our starting point is to assume that the strain energy density $W(\boldsymbol{F})$, the kinetic relation for interfaces, and the nucleation behavior of interfaces, are well-characterized and available. Our approach then takes this information, and formulates a phase-field model that reproduces the elastic response and the kinetic and nucleation behavior of interfaces. The governing equations of our model are as follows.

The energy density has the form:

$$
\left.\stackrel{\circ}{W}^{(\boldsymbol{E}}, \phi\right)=\left(1-H_{l}(\phi-0.5)\right) \psi_{1}(\boldsymbol{E})+\left(H_{l}(\phi-0.5)\right) \psi_{2}(\boldsymbol{E})
$$

where $H_{l}$ is a smooth function that resembles the Heaviside. The functions $\psi_{1}$ and $\psi_{2}$ have the form:

$$
\psi_{A}(\boldsymbol{E})=W\left(\boldsymbol{E}_{A}\right)+\underbrace{\boldsymbol{\sigma}_{T}}_{\left.\equiv \frac{\partial W}{\partial \boldsymbol{E}}\right|_{\boldsymbol{E}_{A}}}:\left(\boldsymbol{E}-\boldsymbol{E}_{A}\right)+\frac{1}{2}\left(\boldsymbol{E}-\boldsymbol{E}_{A}\right): \underbrace{\boldsymbol{C}_{T}}_{\left.\equiv \frac{\partial^{2} W}{\partial \boldsymbol{E} \partial E}\right|_{\boldsymbol{E}_{A}}}:\left(\boldsymbol{E}-\boldsymbol{E}_{A}\right)
$$

$\psi_{A}$ approximates the behavior of $W$ near the states $\boldsymbol{E}_{1}$ and $\boldsymbol{E}_{2}$.

The total energy is then:

$$
\int_{\Omega_{0}}\left(\stackrel{\circ}{W}+\frac{1}{2} \epsilon|\nabla \phi|^{2}\right) d \Omega_{0}
$$

The displacement field evolves through momentum balance:

$$
\operatorname{div}_{\boldsymbol{x}_{0}}\left(\frac{\partial \stackrel{\circ}{ }(\boldsymbol{F}, \phi)}{\partial \boldsymbol{F}}\right)=\rho_{0} \ddot{\boldsymbol{x}}\left(\boldsymbol{x}_{0}, t\right)
$$

In quasistatics, the inertial contribution is set to 0 .

The phase field evolution is governed by the conservation law posed in the companion paper:

$$
|\nabla \phi| v_{n}^{\phi}+G=\dot{\phi}
$$

$G$ is a constitutively-prescribed nucleation term, and $v_{n}^{\phi}$ is the interface velocity field - completely distinct from the material velocity field - through which the kinetic relation is prescribed.

For further details, we refer to the companion paper.

\section{Formulation of a Two-Dimensional Energy for Twinning}

We perform a number of 2D calculations for twinning in the subsequent sections. Here, we outline certain aspects that are common to all those calculations, such as the formulation of $\stackrel{\circ}{W}(\boldsymbol{E}, \phi)$.

The form of $\stackrel{i}{W}$ is:

$$
\stackrel{\circ}{W}(\boldsymbol{E}, \phi)=\left(1-H_{l}(\phi-0.5)\right) \frac{1}{2}\left(\boldsymbol{E}-\boldsymbol{E}_{1}\right): \boldsymbol{C}:\left(\boldsymbol{E}-\boldsymbol{E}_{1}\right)+H_{l}(\phi-0.5) \frac{1}{2}\left(\boldsymbol{E}-\boldsymbol{E}_{2}\right): \boldsymbol{C}:\left(\boldsymbol{E}-\boldsymbol{E}_{2}\right)
$$


Both $\boldsymbol{E}_{1}$ and $\boldsymbol{E}_{2}$ are the stress-free states because $\boldsymbol{\sigma}_{T}=0$. Both wells are at the same height, i.e., $\grave{W}\left(\boldsymbol{E}_{1}, 0\right)=\stackrel{\circ}{W}\left(\boldsymbol{E}_{2}, 1\right)=0$. This is appropriate for twinning, but perhaps not so for other transformations. The relative height of the wells is important because it appears directly in the driving force, and is trivial to change if appropriate.

We have assumed for simplicity that the moduli $C$ are the same in each twin, and additionally assumed that $\boldsymbol{C}$ is isotropic. It should be noted that neither of these assumptions are appropriate in real materials [Cla10, Ka198]. However, it is trivial to incorporate physically-appropriate forms for $\boldsymbol{C}$ in the energy formulated above when required.

For twinning, consider the transformation stretch tensors:

$$
\boldsymbol{U}_{1}=\left[\begin{array}{ll}
\alpha & 0 \\
0 & \beta
\end{array}\right], \boldsymbol{U}_{2}=\left[\begin{array}{ll}
\beta & 0 \\
0 & \alpha
\end{array}\right], \quad \alpha=1-0.1042, \beta=1+0.09659
$$

$\boldsymbol{E}_{1}$ and $\boldsymbol{E}_{2}$ are computed from $\boldsymbol{F}_{1}=\boldsymbol{U}_{1}$ and $\boldsymbol{F}_{2}=\boldsymbol{U}_{2}$. The stress-free compatible interfaces between these wells have normals $\frac{1}{\sqrt{2}}\left(\begin{array}{l}1 \\ 1\end{array}\right)$ and $\frac{1}{\sqrt{2}}\left(\begin{array}{c}1 \\ -1\end{array}\right)$.

In certain cases, we rotate the specimen by $\pi / 12$ radians with respect to the coordinate axes. In that case, the compatible interfaces are oriented with normal $\frac{1}{2}\left(\begin{array}{c}\sqrt{3} \\ 1\end{array}\right)$ and $\frac{1}{2}\left(\begin{array}{c}-1 \\ \sqrt{3}\end{array}\right)$. The reason is that an interface that is inclined at a large angle will feel the effects of the loading on the right boundary of the domain quite differently at different points along its length. On the other hand, an interface aligned perfectly normal to the top and bottom boundaries of the domain will not have any shear stress in the direction tangent to the interface at the junction between the interface and the domain boundary. Some level of shear stress is required for evolution on the boundary/interface junction (Section 10). The angle that we have chosen balances between these competing reasons.

\section{Non-Monotone Kinetic Laws}

Non-monotone kinetic laws have been predicted to show extremely complex and interesting behavior, e.g. [RK97]. However, in that literature, the driving force is taken as a function of interface velocity. In the case where it is non-monotone, it is not invertible to obtain the interface velocity as a function of driving force. In our case, we assume that the interface velocity is a non-monotone function of driving force. We briefly present the results of calculations in 1D and 2D, but the summary is that there is no complex and unexpected behavior as observed in [RK97]. We have verified after the calculations that the the level of driving force was appropriate to access the non-monotonic portion of the kinetic response. 


\subsection{D Non-Monotone Kinetics}

We use the following material model:

$$
\begin{aligned}
\stackrel{\circ}{W}\left(u_{x}, \phi\right) & =\left(1-H_{l}(\phi-0.5)\right) \frac{1}{2} C\left(u_{x}-\varepsilon_{1}\right)^{2}+H_{l}(\phi-0.5) \frac{1}{2} C\left(u_{x}-\varepsilon_{2}\right)^{2} \\
\sigma & =\frac{\partial \stackrel{\circ}{\partial}\left(u_{x}\right)}{=}\left(1-H_{l}(\phi-0.5)\right) C\left(u_{x}-\varepsilon_{1}\right)+H_{l}(\phi-0.5) C\left(u_{x}-\varepsilon_{2}\right) \\
f & =\delta_{l}(\phi-0.5)\left(\frac{1}{2} C\left(u_{x}-\varepsilon_{1}\right)^{2}-\frac{1}{2} C\left(u_{x}-\varepsilon_{2}\right)^{2}\right)+\epsilon \phi_{x x} \\
\rho \ddot{u} & =\sigma_{x} \\
\dot{\phi} & =\left|\phi_{x}\right| v_{n}^{\phi}
\end{aligned}
$$

The stored energy density near each well is quadratic with wells at $\varepsilon_{1}=0$ and $\varepsilon_{2}=1$.

The kinetic response is chosen to be non-monotonic:

$$
\hat{v}_{n}^{\phi}=\left\{\begin{array}{lll}
|f| \cdot(0.1-|f|) & \text { if } \quad|f|<0.075 \\
0.075 \cdot(0.1-0.075) & \text { if } \quad|f| \geq 0.075
\end{array}\right.
$$

Fig. 1 shows the evolution of $\phi$ and $u_{x}$ which are qualitatively similar to the calculations with simpler kinetic response functions.
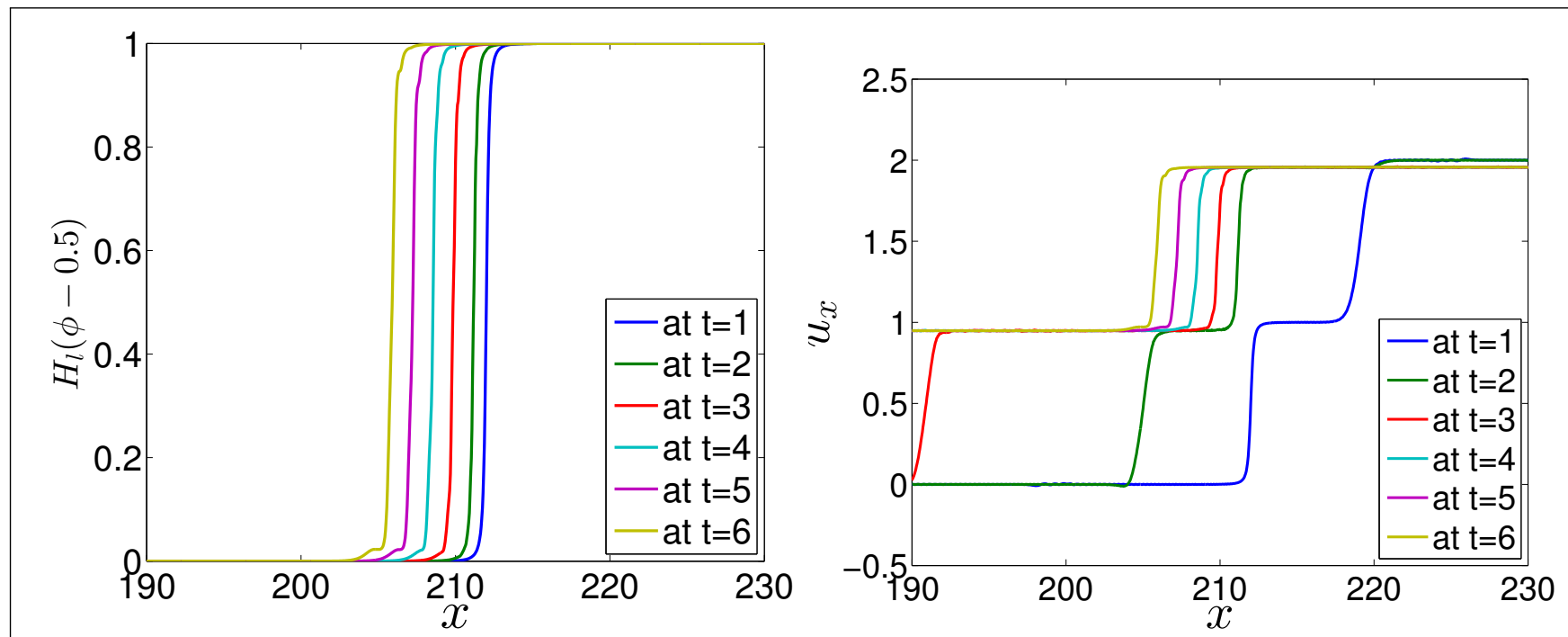

Figure 1: Evolution of $H_{l}(\phi(x)-0.5)$ (left) and $u_{x}$ (right) with a non-monotone kinetic response.

\subsection{D Non-Monotone Kinetics}

We examine 2 settings with non-monotone kinetics; first, a problem with a stress-free compatible interface, and second, where there is necessarily stress around the interface. The reasoning to test both cases is that it is possible that elastic compatibility will dominate the evolution, and therefore testing both cases 
will let us compare the role of kinetics. We compare a linear kinetic response and a non-monotone kinetic response. For the latter, we use the same kinetic response as in 1D from (4.6).

We first examine the case of stressed interfaces using a square plate where a circular region near the center has a second phase. The energy is described in Section 3, and for the incompatible wells we use:

$$
\boldsymbol{U}_{1}=\left[\begin{array}{ll}
1 & 0 \\
0 & 1
\end{array}\right], \boldsymbol{U}_{2}=\left[\begin{array}{ll}
\beta & 0 \\
0 & \alpha
\end{array}\right], \quad \alpha=1-0.1042, \beta=1+0.09659
$$

Fig. 2 shows the evolution through $F_{11}-1$ at various times for the linear and non-monotone kinetic responses respectively. While there are quantitative differences, they are no obvious qualitative differences, and further there is no complex behavior in the non-monotone case.

The previous calculation leaves open the possibility that the kinetics is possibly complex but that momentum balance simply dominates due to the stresses that are necessarily present. Therefore, we briefly examine a problem with a stress-free compatible interface. The energy is described in Section 3, and we use $\boldsymbol{E}_{1}$ and $\boldsymbol{E}_{2}$ as described there, with the rotated sample. We consider a 2D rectangular plate fixed at the left edge, and traction-free at the top and bottom edges. A stress free compatible phase interface exists in the plate initially. A constant tensile load is then applied at the right edge. Fig. 3 shows the initial configuration, and the configuration after some evolution has occurred for both linear and non-monotone kinetics.

\section{Anisotropic Kinetics in Two Dimensions}

We consider the role of anisotropic kinetics in a 2D transformation. To isolate the role of anisotropy in the kinetics, we keep all other effects isotropic. Therefore, we use the energy described in Section 3, but with stress-free wells:

$$
\boldsymbol{U}_{1}=\alpha \boldsymbol{I} \quad \text { and } \quad \boldsymbol{U}_{2}=\beta \boldsymbol{I}, \quad \text { with } \alpha=1.05, \beta=1.1
$$

These wells not stress-free compatible.

We consider a square domain with a hydrostatic loading applied on the boundary. The material is entirely in a single phase, but the new phase nucleates as the loading increases. To force the nucleation to occur away from the boundaries, we make the source term heterogeneous, and of the form:

$$
G(\phi, \boldsymbol{\sigma}, \boldsymbol{x})= \begin{cases}A_{0-1} H_{l}(1-\phi) & \text { if }\left|\boldsymbol{\sigma}_{11}+\boldsymbol{\sigma}_{22}\right|>\sigma_{0} \text { and }|\boldsymbol{x}|<0.1 \\ 0 & \text { otherwise }\end{cases}
$$

$A_{0-1}$ is a constant characteristic of the $0-1$ reaction, and $H_{l}(1-\phi)$ ensures that the 1 -phase is created only when we are not already in the 1-phase, i.e., the nucleation term turns off when the 1-phase has nucleated. The nucleation term is only active in a circle centered around the middle of the domain, and is only active when the hydrostatic stress $\left|\boldsymbol{\sigma}_{11}+\boldsymbol{\sigma}_{22}\right|$ is above a critical stress $\sigma_{0}$. We have not allowed for the reverse transformation, but it is trivial to add this if required. The nucleation term and the loading both maintain the circular symmetry in the problem.

The kinetic response is $\hat{v}_{n}^{\phi}(f)=\operatorname{sign}(f) \kappa|f|\left|\frac{\nabla \phi}{|\nabla \phi|} \cdot \boldsymbol{d}_{m}\right|$, where $\boldsymbol{d}_{m}=\frac{\sqrt{3}}{2} \boldsymbol{e}_{1}+\frac{1}{2} \boldsymbol{e}_{2}$ is a distinguished material direction that sets the anisotropy. Recall that $\frac{\nabla \phi}{|\nabla \phi|}$ sets the normal to the interface. Therefore, the 


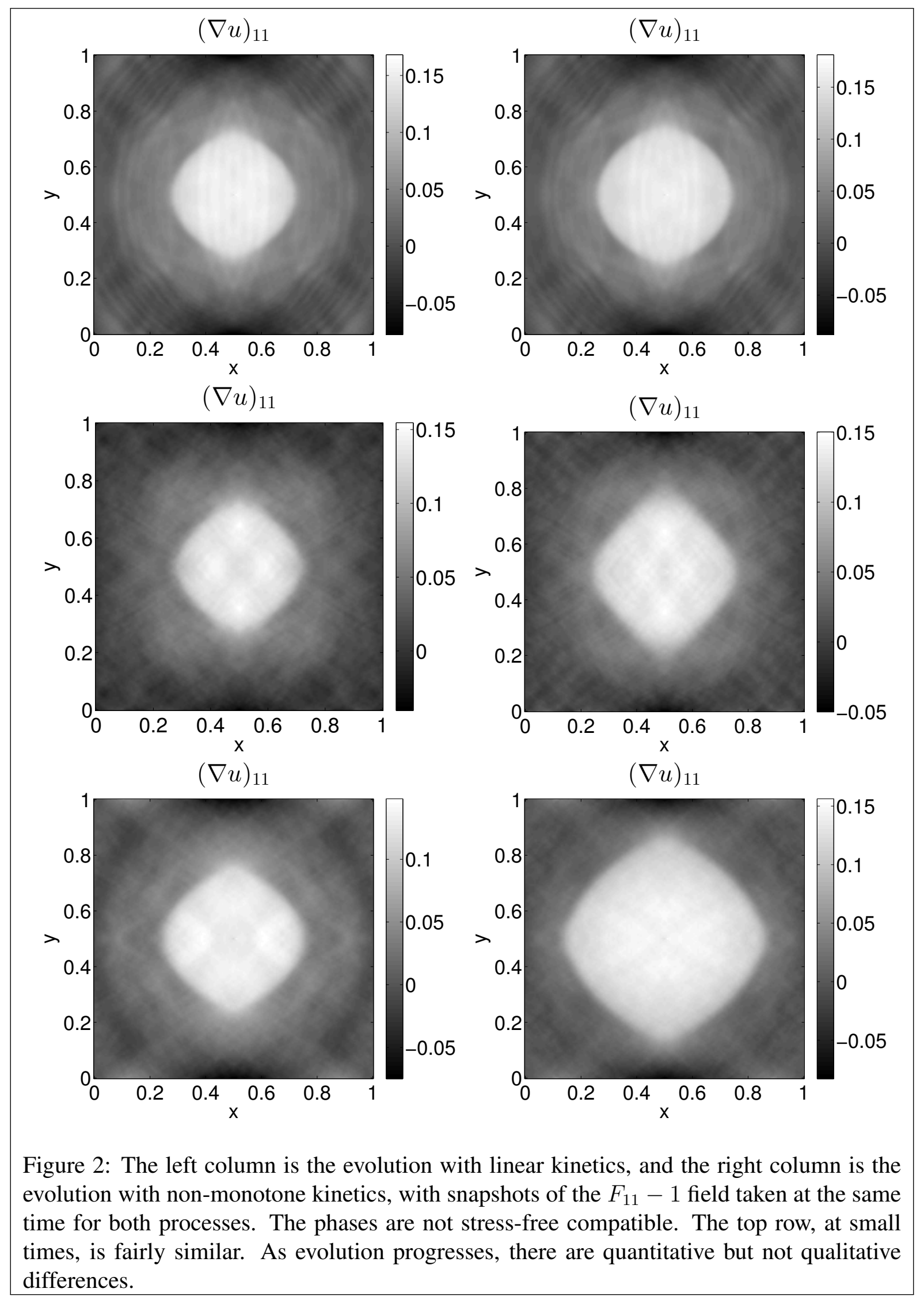



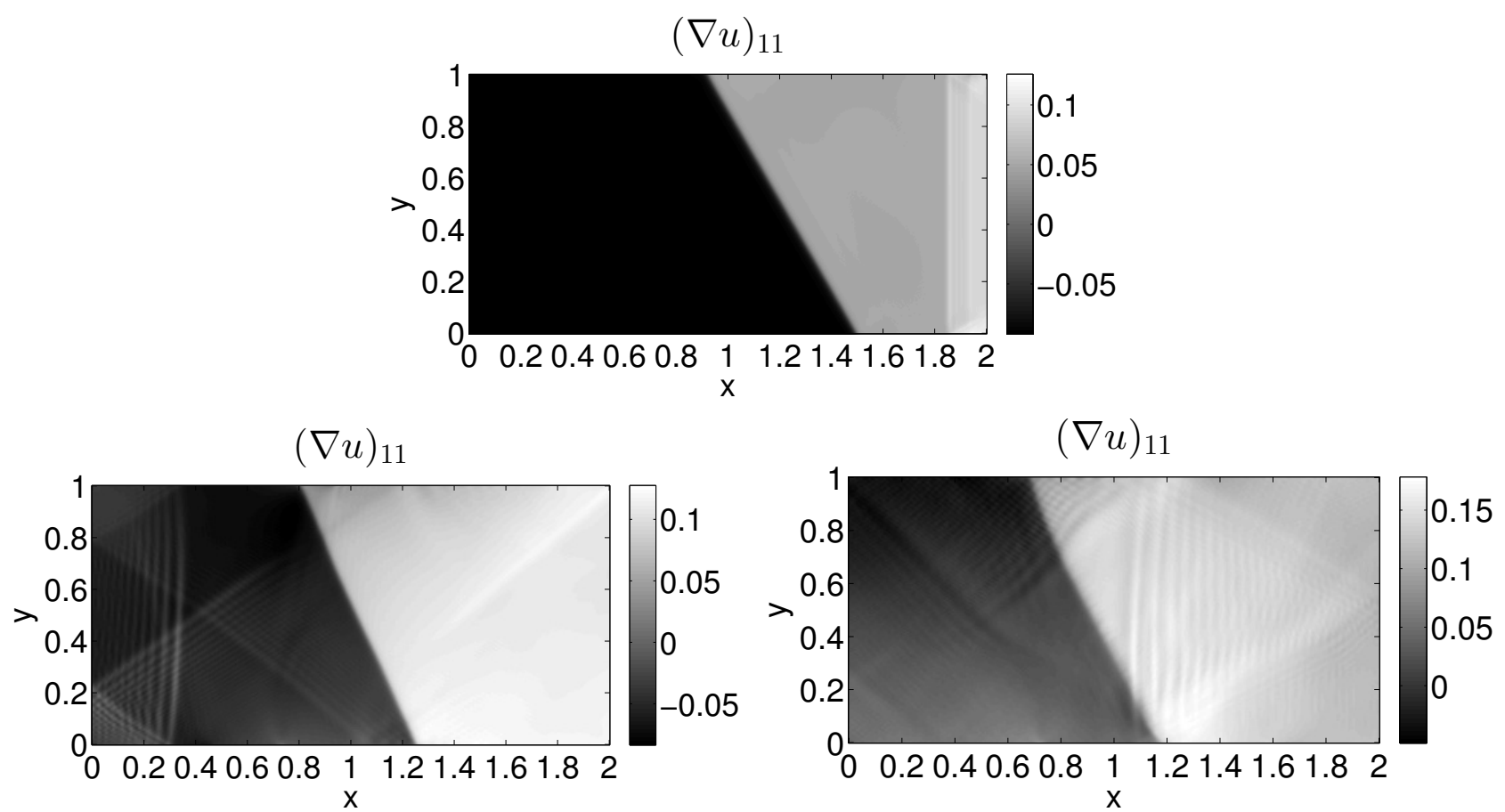

Figure 3: The top figure shows the initial configuration, the lower left figure shows the configuration after some time using linear kinetics, and the lower right figure shows the configuration after some time using non-monotone kinetics. All plots are of the $F_{11}-1$ field. As in the incompatible case, there are quantitative but no obvious qualitative differences.

kinetic response depends on the relative orientation of the interface to $\boldsymbol{d}_{m}$, and interface velocity goes to 0 along directions normal to $\boldsymbol{d}_{m}$.

The evolution of the deformation is shown in Fig. 4.

\section{Stick-slip Twinning Kinetics}

We examine the evolution of twinning interfaces in 2D using stick-slip kinetics using the energy described in Section 3 with the rotated specimen. We consider a 2D rectangular plate fixed at the left edge, and traction-free at the top and bottom edges. A stress-free compatible phase interface exists in the plate initially. A constant tensile load is then applied at the right edge.

We use a stick-slip kinetic response: $\hat{v}_{n}^{\phi}=0$ if $|f|<f_{0}$ and $\hat{v}_{n}^{\phi}=\kappa \operatorname{sign}(f)\left(|f|-f_{0}\right)$ if $|f| \geq f_{0}$. We examine the evolution of the interface for a number of applied load levels. Fig. 5 shows the $F_{11}-1$ field some time after evolution has commenced, for different load levels. We find that we are able to impose stick-slip easily and effectively through the kinetic response listed above in $2 \mathrm{D}$ as well. 


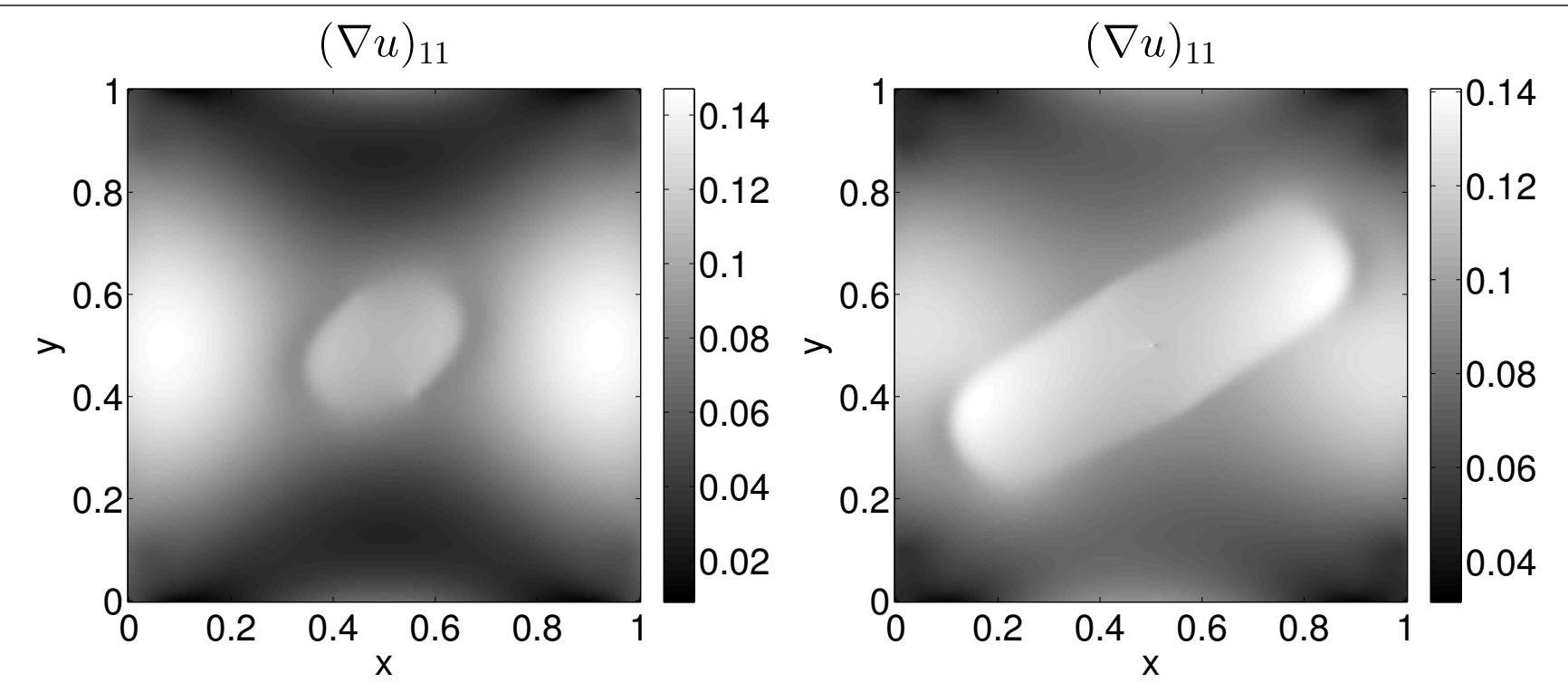

Figure 4: The $F_{11}-1$ field at different times for an anisotropic kinetic response. Nucleation takes place within a circle of radius 0.1 at the center of the domain. The interface velocity is anisotropic, and the effect can clearly be seen because everything else in the problem maintains circular symmetry. It can be seen that regions where the interface normal is perpendicular to $\boldsymbol{d}_{m}$ do not show any motion / growth after the initial nucleation stage.

\section{Rate-Dependent and Asymmetric Nucleation}

We examine the prescription of complex nucleation rules in our formulation. In this section, we demonstrate two key features: (1) that we transparently incorporate complex nucleation behavior such as ratedependence; and (2) that we can tailor the nucleation stress easily without any modifications to the energy but purely through the activation of the source term $G$. We perform calculations in 1D and 2D to show the ability of the model to separate kinetics and energetics from nucleation. An additional feature that we demonstrate is that we can independently prescribe the forward and reverse nucleation stresses; i.e., given a completely symmetric energy landscape, we are able to induce nucleation in one direction at a certain critical stress, but the reverse transformation is induced at a completely independent critical value of the stress.

This gives us a powerful approach to prescribe complex nucleation criteria. For instance, in the 1D calculation described below, we are able to change the nucleation stress by a factor of 3 for a change of $4 \%$ in the loading rate.

\subsection{Rate-Dependent and Asymmetric Nucleation in One Dimension}

We use the same energy $\stackrel{\circ}{W}$ as in Section 4.1 with linear kinetics. This energy is quadratic around the stress-free strains 0 and 1, corresponding to phase 1 and phase 2 . The elastic moduli is the same in both phases. 

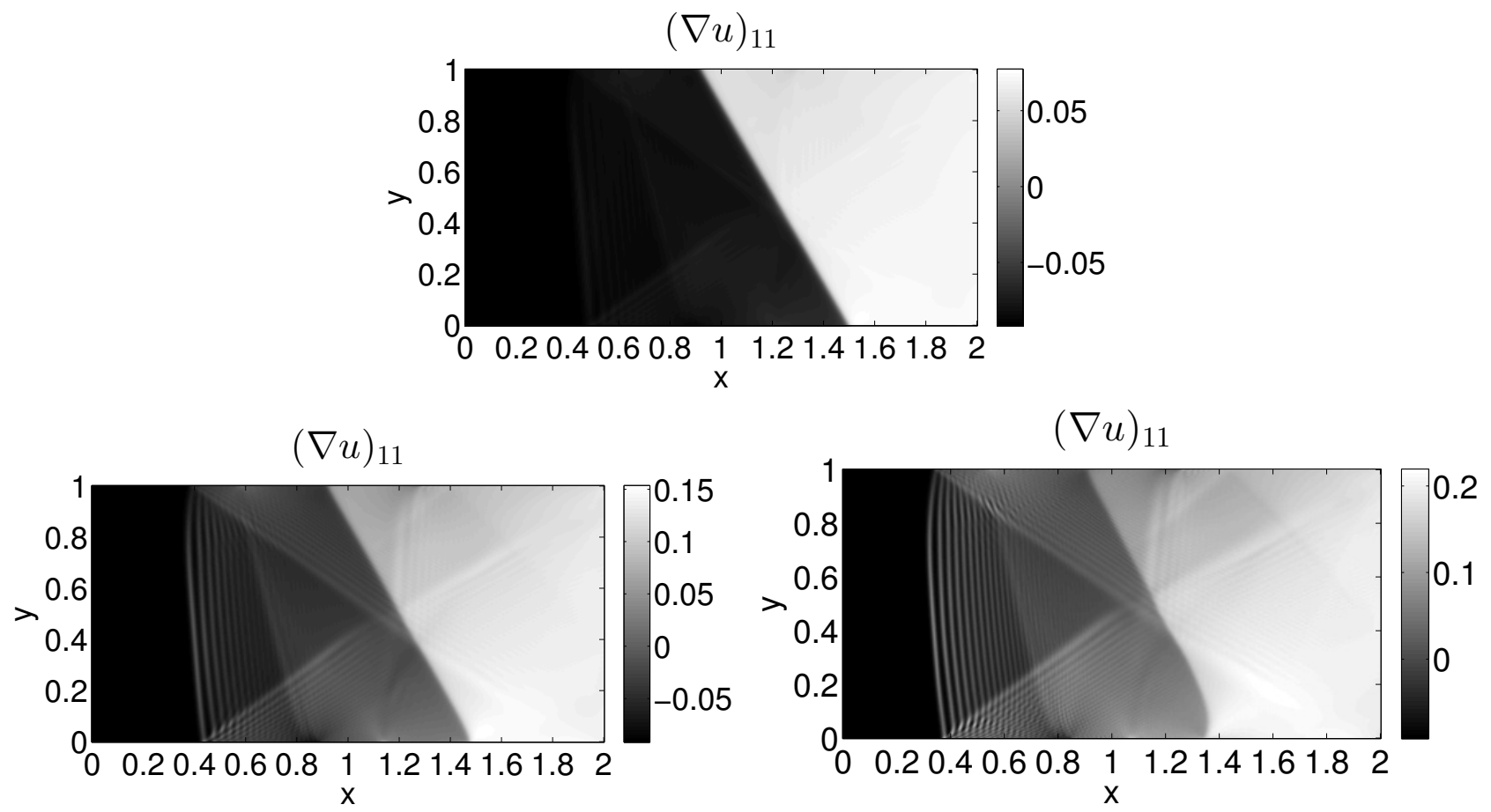

Figure 5: The $F_{11}-1$ field at a given time for varying applied load levels. In the first case, well below the critical load, there is no evolution; in the second case, just above the critical load, there is very slow evolution; in the third case, well above the critical load, there is rapid evolution.

The nucleation criterion is specified as follows:

$$
G(x)= \begin{cases}\left(1-H_{l}(\phi-0.6)\right) & \text { if } \sigma(x)>\sigma_{1 \rightarrow 2} \\ H_{l}(\phi-0.4) & \text { if } \sigma(x)<\sigma_{2 \rightarrow 1} \\ 0 & \text { else }\end{cases}
$$

The term $\left(1-H_{l}(\phi-0.6)\right)$ ensures consistency with thermodynamics in that it turns off the nucleation source for the transformation $1 \rightarrow 2$ when we are in phase 2. Similarly, $H_{l}(\phi-0.4)$ turns off the source for the reverse transformation $2 \rightarrow 1$ when we are in phase 1 .

For clarity of presentation, we have simplified the nucleation definitions to be piece-wise. However, this would require us to track precisely the point at which the stress reaches the critical value. Therefore, in implementation, we use a regularized version of these definitions, both in this section and elsewhere. The key distinction between the regularization that we have used in the nucleation and kinetics, vs. the usual regularization of displacement fields, is that in our case the observed nucleation and kinetics are not sensitive to the regularization.

Asymmetry in the forward and reverse transformations is readily prescribed by using different values for the forward and reverse threshold stresses $\sigma_{1 \rightarrow 2}$ and $\sigma_{1 \rightarrow 2}$.

Rate-dependence is prescribed by making the threshold stresses functions of the loading rate. We use:

$$
\sigma_{1 \rightarrow 2}=\left\{\begin{array}{ll}
0.06 & \text { if } \dot{\varepsilon}<5.1 \times 10^{-4} s^{-1} \\
0.2 & \text { if } \dot{\varepsilon} \geq 5.1 \times 10^{-4} s^{-1}
\end{array} \quad \text { and } \sigma_{2 \rightarrow 1}= \begin{cases}-0.03 & \text { if } \dot{\varepsilon}<5.1 \times 10^{-4} s^{-1} \\
-0.1 & \text { if } \dot{\varepsilon} \geq 5.1 \times 10^{-4} s^{-1}\end{cases}\right.
$$


We demonstrate this nucleation criterion by computing an entire hysteresis loop, starting with the forward transformation from phase 1 to phase 2, and then the reverse transformation back to phase 1 (Fig. 6). This loop is computed by simply evolving the kinetic equation for $\phi$ while solving the quasistatic (i.e., without inertia) momentum balance as the evolution proceeds.
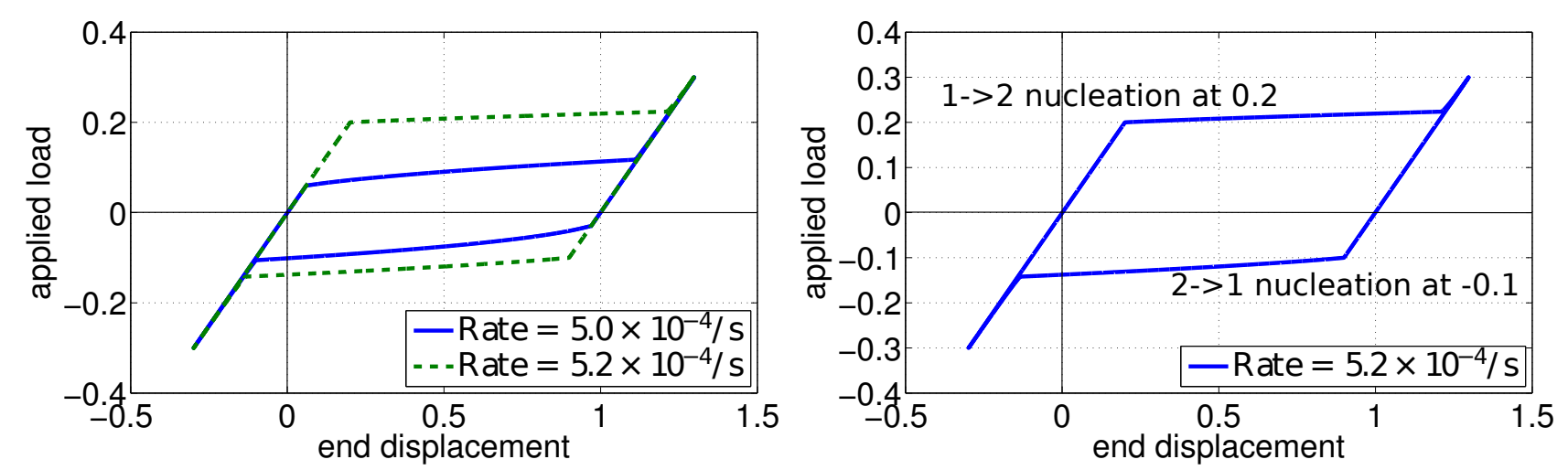

Figure 6: Left: Hysteresis curve for two loading rates demonstrating effect of rate-dependent nucleation. Right: Hysteresis curve demonstrating asymmetric nucleation, i.e., forward and reverse nucleation criteria are independently prescribed without changing the energy ${ }^{\circ}$. The bold horizontal line marks the stress around which the energy $\mathscr{I}^{\circ}$ is symmetric.

As we notice from Fig. 6, we are able to precisely set the nucleation stress in a direct and transparent manner. In addition, we are able to change the nucleation stress by a factor of 3 for a change in loading rate of $4 \%$.

\subsection{Rate-Dependent and Asymmetric Nucleation in Twinning}

We demonstrate here the possibility of allowing rate-dependence and asymmetry in nucleation for a twinning transformation. While twinning is typically expected to be symmetric, this calculation serves as a demonstration that asymmetry in nucleation can be readily introduced in $2 \mathrm{D}$ even when eveything else in the problem is symmetric.

We use the energy for twinning described in Section 3 with linear kinetics. The nucleation criterion is specified as follows:

$$
G= \begin{cases}\left(1-H_{l}(\phi-0.8)\right) & \text { if } \boldsymbol{a} \cdot \boldsymbol{\sigma} \boldsymbol{b}>\sigma_{1 \rightarrow 2} \\ H_{l}(\phi-0.2) & \text { if } \boldsymbol{a} \cdot \boldsymbol{\sigma} \boldsymbol{b}<\sigma_{2 \rightarrow 1} \\ 0 & \text { else }\end{cases}
$$

It resembles closely the $1 \mathrm{D}$ version. The key difference from $1 \mathrm{D}$ is that the scalar stress is here replaced by the shear traction magnitude on the planes oriented at $\pi / 12$. Hence, this is essentially a critical resolved shear stress (CRSS) type of nucleation criterion, with the difference being that we can set $\sigma_{2 \rightarrow 1}$ and $\sigma_{1 \rightarrow 2}$ to completely distinct values.

Asymmetry and rate-dependence are prescribed by following exactly the 1D approach of making $\sigma_{2 \rightarrow 1}$ and $\sigma_{1 \rightarrow 2}$ different from each other and rate-dependent. For simplicity, we set the rate-dependence 
through the 11-component of the nonlinear strain measure. For realistic calculations, a model based on an objective rate would be appropriate.

Fig. 7 shows the hysteresis loop that demonstrates both asymmetry and rate-dependence of our evolution law.

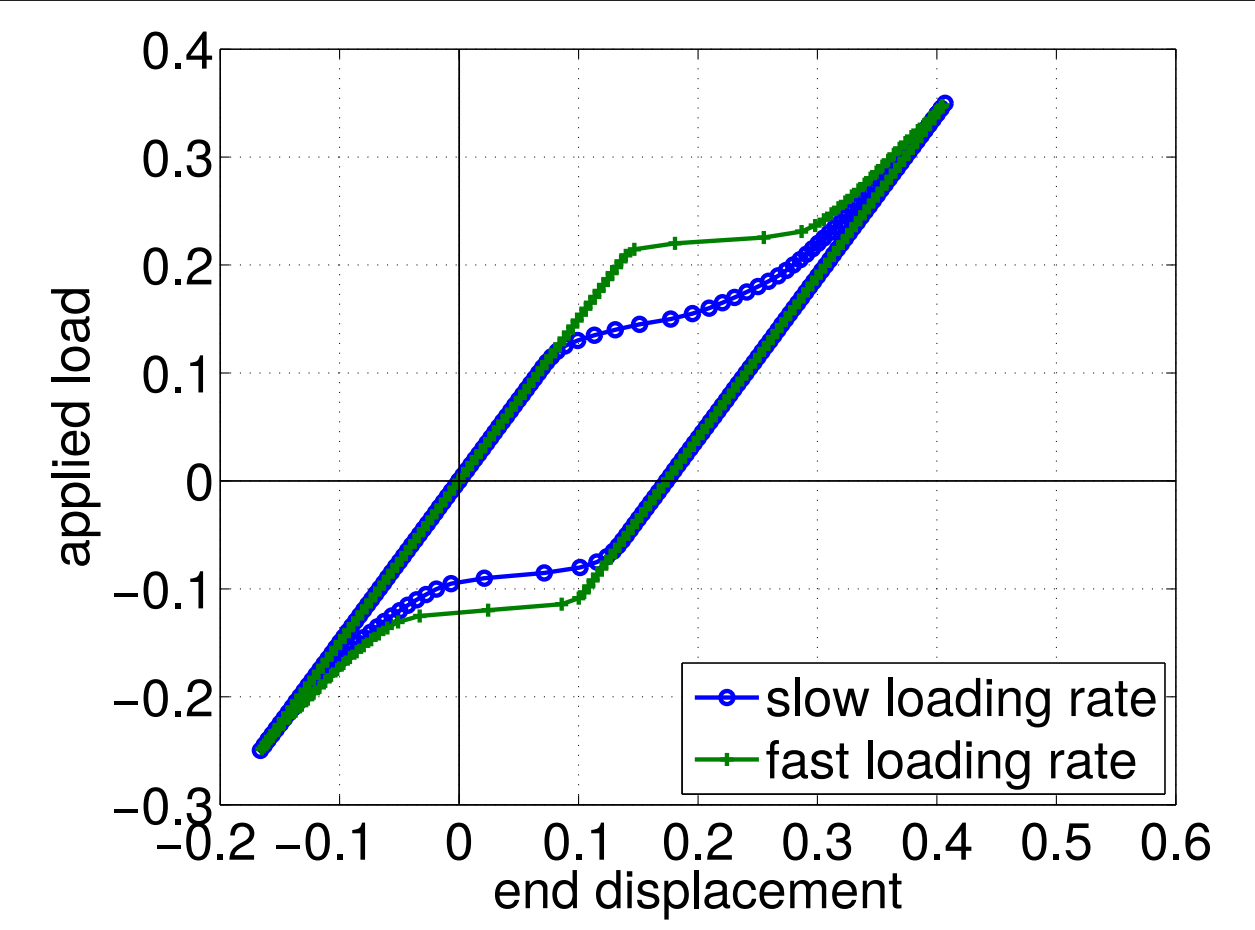

Figure 7: A hysteresis loop for twinning demonstrating the ability to apply rate-dependent and asymmetric nucleation criteria in 2D. For computational efficiency, we have only computed the nucleation points (in the vicinity of the circles), and interpolated between them.

\section{Imposing Principal Normal Stress- and Shear Stress- Dependent Nucleation for Twinning}

Defect nucleation and kinetics typically do not depend exclusively on the driving force. E.g., recent studies of dislocation motion in BCC metals show that other stress components can be important in addition to the shear-related driving force [GBV08, GRBV08]. Motivated by these studies, we examine twin nucleation within our model, where the nucleation criterion has a dependence on the maximum principal stress, in addition to the critical resolved shear stress (CRSS) for twin nucleation. While this nucleation criterion is not motivated by any specific observations in experiments or atomistics, it serves as a toy model to demonstrate the transparent specification of complex nucleation criteria.

The elastic energy is as described in Section 3, with the unrotated specimen. We use simple linear 
kinetics. Our nucleation criterion is as follows:

$$
G(\phi, \boldsymbol{\sigma})= \begin{cases}A_{0 \rightarrow 1} H_{l}(0.6-\phi) & \text { if }|\boldsymbol{b} \cdot \boldsymbol{\sigma} \boldsymbol{a}|>\tau_{C R S S} \text { and } \max \left\{\left|\sigma_{\text {principal }}\right|\right\}>\sigma_{0} \\ 0 & \text { otherwise }\end{cases}
$$

$A_{0 \rightarrow 1}$ is a constant which controls the rate of nucleation. $H_{l}(0.6-\phi)$ causes nucleation to be active when $\phi<1$ and "turns off" when $\phi$ is close to 1 . To prevent nucleation near the boundaries, $G$ is active only in a circle of radius 0.35 centered at the middle of the domain and 0 elsewhere.

The CRSS character is built into the nucleation stress through the condition $|\boldsymbol{b} \cdot \boldsymbol{\sigma} \boldsymbol{a}|>\tau_{C R S S}$. The vectors $\boldsymbol{a}=\frac{1}{\sqrt{2}}\left(\begin{array}{l}1 \\ 1\end{array}\right)$ and $\boldsymbol{b}=\frac{1}{\sqrt{2}}\left(\begin{array}{c}-1 \\ 1\end{array}\right)$ are the twin normal and shear direction, thereby resolving the shear stress in the direction appropriate for twin nucleation. We note that it is direct and transparent to have the nucleation stress have CRSS character as in the equation. It is equally direct to add a dependence on the hydrostatic stress state if required; in our example, we have taken a simple dependence that requires a minimum level of the principal stress. More complex pressure-sensitive nucleation responses can be easily incorporated in our model.

We examine the behavior of this nucleation response through the interaction of an elastic wave with the residual stress field around an inclusion. A square 2D single-phase domain is considered with a circular non-transforming elastic inclusion at the center. The elastic inclusion is stress-free at $\boldsymbol{F}=\boldsymbol{I}$ but the surrounding matrix has $\boldsymbol{F}=\boldsymbol{U}$; therefore, the interface between the inclusion and the matrix sets up a stress field. A shear at - or above - the critical level required by the CRSS criterion would not cause nucleation unless there is also sufficiently large principal stress. The residual stress field around the misfitting inclusion provides this stress.

The specimen is fixed on the left boundary, traction-free at the top and bottom, and a constant load is applied to the right. This load results in an elastic wave which interacts with the stress field around the inclusion and leads to nucleation when the critical conditions are satisfied.

The inclusion is elastic in the sense that it has a finite elastic modulus. However, it has only a single stress-free deformation state at $\boldsymbol{F}=\boldsymbol{I}$. Therefore, $\mathscr{\circ}^{\circ}$ in the inclusion is independent of $\phi$, and the energy has only the term $\frac{1}{2} \epsilon|\nabla \phi|^{2}$ that depends on $\phi$. Therefore, the evolution of $\phi$ is diffusion-driven within the inclusion, and interacts with the matrix through the value of $\phi$ on the boundary. Physically the evolution of $\phi$ within the inclusion has no meaning, but it nonetheless affects the evolution in the matrix through the interaction on the inclusion-matrix boundary. Therefore, the ideal strategy would be to not define $\phi$ at all within the inclusion and use a separate boundary kinetic equation that reflects the physics of the interface interacting with the inclusion-matrix boundary (see Section 10). A simpler approach may be to define the kinetics within the inclusion in such a way as to mimic the correct physics at the inclusion-matrix boundary. For now, we have ignored these issues and simply evolved $\phi$ based on $\frac{1}{2} \epsilon|\nabla \phi|^{2}$.

\section{Competition Between Nucleation and Kinetics in Twinning}

We examine the competition between kinetics and nucleation in the evolution of microstructure. We use precisely the same material model, specimen geometry and inclusion, and loading conditions as in Section 8. Recalling that $G$ is a nucleation rate, we examine the effect of a large nucleation rate and a small nucleation rate, while keeping the kinetic response the same in both cases. Varying the nucleation rate simply involves changing the value of $A_{0 \rightarrow 1}$ in (8.1). 

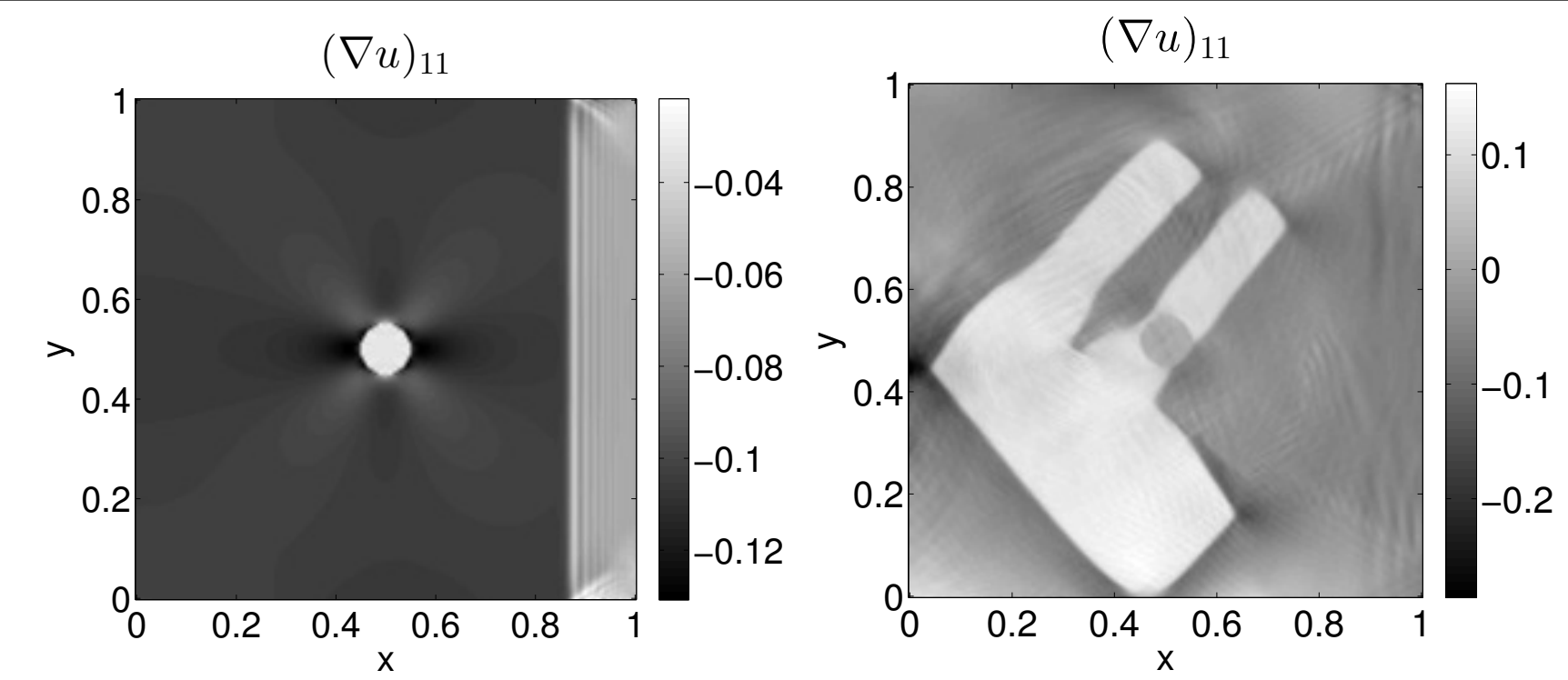

Figure 8: The $F_{11}-1$ field before the elastic waves interact with the stress field around the circular inclusion (left), and after (right). Complex nucleation patterns can be seen, with the elastic stresses driving the interfaces - through the driving force in the kinetic response - to incline at $\pm \pi / 4$. The lighter regions indicate the nucleated phase. Elastic waves that are created during the process of nucleation are also seen.

The results of the calculations are shown in Fig. 9. A key qualitative difference is that when the nucleation rate is smaller, we find that the microstructure is finer as compared to the larger nucleation rate. Heuristically, it appears that the high nucleation rate enables the formation of larger nuclei that quickly coalesce to form relatively coarse-microstructure. For lower nucleation rates, the microstructure takes longer to develop and there are a number of smaller nuclei. We note that the total area of the transformed regions is smaller when the nucleation rate is lower, as may be expected.

\section{Boundary Kinetics}

The most widely-used boundary condition (BC) on $\phi$ in standard phase-field modeling imposes $\nabla \phi \cdot N=$ 0 on $\partial \Omega$. Among other things, it forces the interface to be normal to the boundary in the vicinity of the boundary, and it does not associate a kinetics to the evolution on the boundary beyond that which is driven by the evolution in the bulk. However, as [SB98, SB00, SB99] examined in the sharp-interface setting, it is possible to associate a separate kinetics to the junction line (or junction point in 2D) of the interface with the boundary. Along the junction line, we require not only the normal interface velocity field $v_{n}^{\phi}$, but also the interface velocity component that is tangent to the boundary. As illustrated in Fig. 10 , the boundary velocity can have a profound effect on the evolution on the interior. It also controls the surface evolution of $\phi$, and ignoring the effect of boundary kinetics can potentially lead to errors in analyzing experiments that are based only on surface measurements.

In this section, we use the interface balance principle to deduce a kinetic law for the regularized junction lines, and apply the formulation to some illustrative calculations. 


\subsection{Formulation of Boundary Kinetics}

Following closely the balance principle posed in the companion paper, we consider the flux of interfaces that thread a curve, with the only difference being that in this case the curve is restricted to lie on the boundary. Hence, the kinetic law is the same as the bulk, $\dot{\phi}=|\nabla \phi| v_{e}^{\phi}+G_{e}$, with the gradient operator here interpreted as restricted to the boundary. As with $v_{n}^{\phi}$, the edge velocity $v_{e}^{\phi}$ can be an arbitrary function of any arguments as long as it satisfies positive dissipation. To ensure positive dissipation, as well as identify the thermodynamic conjugate driving force, we re-examine the dissipation statement, but augment the energy to account for the boundary working.

We start by rewriting the energy to include an "elastic energy on the boundary":

$$
\int_{\Omega_{0}} \stackrel{\circ}{W}(\boldsymbol{F}, \phi)+\int_{\Omega_{0}} \frac{1}{2} \epsilon|\nabla \phi|^{2} \boldsymbol{\alpha}+t \int_{\partial \Omega_{0}} \stackrel{\circ}{W}(\boldsymbol{F}, \phi)+\int_{\partial \Omega_{0}} \frac{1}{2} \epsilon_{S}\left|\nabla_{S} \phi\right|^{2}
$$

$\nabla_{S}$ is the gradient on the surface.

The first surface integral is the "elastic energy on the boundary". The constant $t$ that multiplies it has dimensions of length, and is required for dimensional consistency. Heuristically, it can be considered a measure of the distance that the surface effects penetrate into the bulk.

The term containing $\epsilon_{S}$ regularizes the interface-boundary junction. In principle, it is possible for the elastic energy on the boundary to drive the interface to become singularly sharp at the junction, and this term prevents that. Physically, it allows for the junction to have a width that can differ from the width of interfaces in the bulk. We use $\epsilon_{S}$ to define $t \sim \epsilon_{S} / \epsilon$ which has dimensions of length.

For notational convenience, we write the energy in (10.1) as $V[\boldsymbol{u}, \phi]+S[\boldsymbol{u}, \phi]$, where $V$ consists of the volume integrals and $S$ consists of the surface integrals. We write the kinetic energy also as a sum of a volume integral and surface integral:

$$
K=K_{v}+K_{s}=\int_{\Omega_{0}} \frac{1}{2} \rho \boldsymbol{V} \cdot \boldsymbol{V}+t \int_{\partial \Omega_{0}} \frac{1}{2} \rho \boldsymbol{V} \cdot \boldsymbol{V}
$$

We now examine dissipation to obtain the thermodynamic conjugate driving forces for the the bulk and the edge interface velocities. Recall that $\mathcal{D}$ is the difference between rate of external working and the rate of change of stored energy. Computing $\mathcal{D}$ gives us precisely the same result as the dissipation calculations in the companion paper, except that we have the additional contribution from the surface terms in the stored energy. This additional contribution is $-\frac{d}{d t}\left(S[\boldsymbol{u}, \phi]+K_{s}\right)$, and can be simplified as follows:

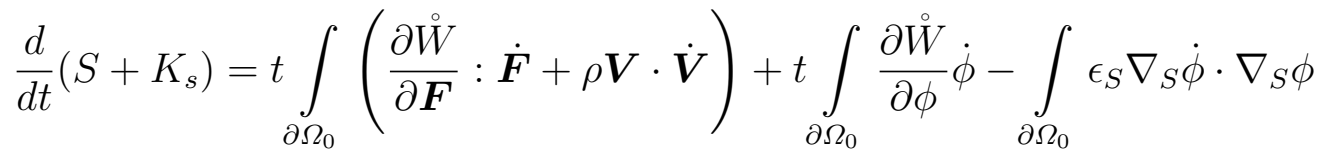

The first term above vanishes as follows. Following precisely the calculations in the companion paper, we can write $\dot{F}$ as the material velocity gradient. Using integration-by-parts and the divergence theorem, we obtain balance of linear momentum which consequently vanishes, and another term that is a total derivative integrated over the closed region $\partial \Omega_{0}$ and therefore is set to 0 .

The last term above can be written as $\int_{\partial \Omega_{0}} \epsilon_{S} \dot{\phi} \operatorname{div}_{S} \nabla_{S} \phi$, as well as another term that is a total derivative integrated over the closed region $\partial \Omega_{0}$ and therefore goes to 0 . 
We can now write the dissipation $\mathcal{D}$. It consists of the same expressions as in the dissipation without bulk contributions (see companion paper), and with the additional remaining nonzero terms from (10.3):

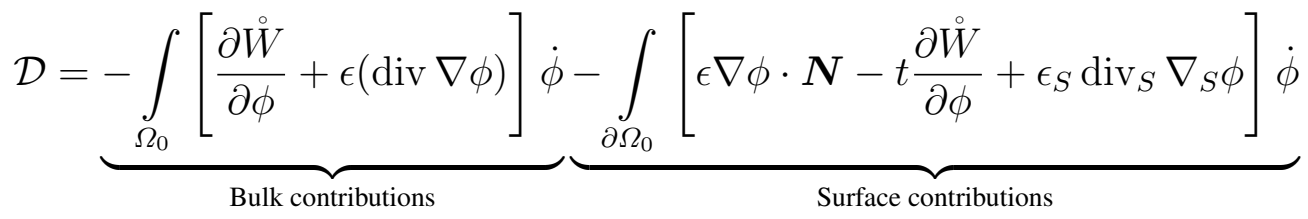

We use the bulk and boundary balance laws to replace $\dot{\phi}$ by $|\nabla \phi| v_{n}^{\phi}$ and $|\nabla \phi| v_{e}^{\phi}$ to obtain the thermodynamic conjugates to $v_{n}^{\phi}$ and $v_{e}^{\phi}$ as the bulk and edge driving forces:

$$
f_{\text {bulk }}:=-\frac{\partial \stackrel{\circ}{W}}{\partial \phi}+\epsilon \operatorname{div} \nabla \phi, \quad f_{\text {edge }}:=-t \frac{\partial \stackrel{\circ}{W}}{\partial \phi}+\epsilon_{S} \operatorname{div}_{S} \nabla_{S} \phi-\epsilon \nabla \phi \cdot \mathbf{N}
$$

The expression for $f_{\text {edge }}$ has some similarities to the sharp-interface version [SB98], in much the same way as $f_{b u l k}$ has similarities to the classical driving force on an interface.

We have ignored the source $G^{e}$ above, but it provides a natural avenue to control nucleation of new interfaces on the boundary.

\subsection{Faceting of a Flat Stressed Interface}

We consider the energy in Section 3 using the unrotated specimen. As noted there, the stress-free compatible twin interfaces are oriented at $\pm \pi / 4$. Here, we begin with an interface oriented vertically, and examine the evolution driven by the stress field. As expected, the interface facets to locally align along the stress-free compatible directions. However, we examine the effect of two contrasting choices of the boundary kinetics: (1) linear boundary kinetics that matches the linear bulk kinetics, and (2) pinned on the boundary with linear bulk kinetics. We fix the left face of the specimen, and leave the other faces traction-free.

Fig. 11 shows the initial condition for both cases, and the final equilibrium state. An interesting result when the interface is pinned on the boundary is that the orientation of the interface at the boundary is exactly the opposite of the interface orientation in the bulk. Experimental probes of microstructure at the surface could completely mis-estimate the bulk microstructure.

\subsection{Competition Between Bulk and Boundary Kinetics}

We compare the evolution of a stress-free compatible interface for 3 different cases: when the boundary kinetics is (i) slower, (ii) the same, and (iii) faster, than the bulk kinetics. For both bulk and boundary kinetics, we use a linear dependence on driving force, and change only the leading coefficient multiplying them. The ratios of the coefficients are 0.4 (boundary slower than bulk), 1.0 (boundary and bulk are the same), and 1.6 (boundary faster than bulk).

We use the material described in Section 3 with the rotated specimen. The initial configuration with the stress-free compatible interface for all 3 cases is the same and shown in Fig. 12. A constant tensile load is applied at the right face of the domain, and this causes the interface to begin to move. The top and bottom faces are traction-free, and the left end is clamped. The results from the 3 cases are shown in Fig. 12. 


\subsection{A Singularity In Boundary Kinetics}

The simplest boundary kinetic relation is to assume that the edge velocity field $v_{e}^{\phi}$ is a function of only the driving force $f_{\text {edge }}$. However, we discuss here a simple but realistic example where this can lead to unexpected and likely-unphysical results.

Consider the energy in Section 3 for twinning, but with the specimen oriented such that the stress-free twin interface is vertical. The interface is normal to the boundary on the top and bottom faces which are traction-free. We apply a shear force on the right face while the left face is held fixed.

These phases in this problem are related by a shear, and hence the driving force for transformation is directly proportional to the shear traction on the plane that is parallel to the interface. Therefore, a simple choice of linear kinetics - in the bulk as well as on the boundary - will set the interface velocity roughly proportional to the local value of the shear traction on the interface.

However, the top and bottom faces of the domain are traction-free; therefore, the shear stresses along the interface at the top and bottom faces are 0 . The boundary driving force then largely vanishes, except for a small contribution due to the regularization parameters $\epsilon$ and $\epsilon_{S}$. Fig. 13 shows the time evolution of the interface in the vicinity of the boundary. We find the unexpected development of an extremely curved interface as the interface in the interior moves forward while the interface-boundary junction barely moves. The presence of $\epsilon$ and $\epsilon_{S}$ prevent this extremely curved interface from becoming completely singular. With this regularization, the interface moves slowly along the boundary as the large curvature leads to contributions from the higher-order derivatives.

While this regularization keeps the problem from becoming singular, it does not seem physically attractive for the evolution to be dominated by ad-hoc regularization terms. An approach that may perhaps provide more physically-meaningful evolution is to set the boundary kinetics based on $\nabla \phi \cdot \boldsymbol{N}$; recall that the typical boundary condition in standard phase-field methods is $\nabla \phi \cdot N=0$. However, as pointed out previously, this will drive the interface to be normal to the boundary in the vicinity of the boundary.

\section{Discussion}

We have presented the 2D characterization of a phase-field model - formulated in the companion paper - that has non-singular interfaces yet allows for transparent prescription of kinetics and nucleation of interfaces. A number of examples displaying complex kinetics and nucleation are seen to be easily and transparently attacked by our approach. We have also extended the phase-field framework to allow for the prescription of an independent kinetics for the junction line between an interface and a material surface.

Our formulation allows us to prescribe both kinetics and nucleation independently. From various numerical calculations, we find that microstructure patterns and mechanical response can be very sensitive to the kinetics, particularly for stick-slip and anisotropic kinetics. Both stick-slip and anisotropic kinetics are key elements in many physical situations of interest. Standard phase-field does not handle stick-slip well when it is imposed by simply stopping the evolution when the driving force is below some critical value [LLP10], but it is unclear if this difficulty is due to the energy structure or the evolution law. Non-monotone kinetic relations, however, were found not to provide any complex behavior.

We find that nucleation plays an extremely significant role in the development of microstructure patterns and mechanical response. We have presented a number of examples to show the transparent and easy 
prescription of complex nucleation rules in our formulation. These nucleation rules can involve various components of stress and strain, their rates, nonlocal quantities, and so on. We emphasize that the nucleation criteria that we have used are toy models to enable the demonstration that we can readily and transparently incorporate an extremely broad range of nucleation criteria. In addition to enabling calibration to experiment or atomic calculations, this capability enables us to systematically probe the roles of different physical mechanisms for nucleation. Recently, [BT10, WBT10] presented a first-principles-motivated statistical model of twin nucleation in HCP materials. While we have only examined deterministic nucleation in this work, it is straightforward to replace the deterministic source term in our evolution law by a statistical object that incorporates the insights from [BT10, WBT10].

An important work with an alternative approach to the goal of numerical simulations of interface kinetics and nucleation is [HRL99]. They use a level-set like approach but with a careful approach to regularization that aims to preserve the sharp-interface kinetics. While there are many similarities between our work and theirs, there are also some key differences. In our approach, we aim to formulate a model with certain key features such as regularized interfaces and the ability to prescribe nucleation and kinetics. Their approach, however, is to develop a careful and sophisticated numerical method that reproduces the sharp-interface model. A further difference appears to be the relative ease of numerical implementation of our model compared to that approach, but this could change.

Phase-field methods have largely been restricted to linear elastic models. This has the advantage that the elastic problem has nice properties such as existence, uniqueness etc. While we have used a nonlinear deformation model in some of our calculations, it is convex (and quadratic) in the nonlinear strain for a fixed value of $\phi$; we note that convexity in the nonlinear strain measure chosen here does not guarantee these nice properties [Cia88]. Other recent phase-field models with large-deformation elasticity include [PL13, CK11, Lev14].

Our extension to incorporate boundary kinetics provides an interesting contrast with standard phase-field models. In those models, the phase-field evolution has roughly the character of a diffusion equation if one considers the highest derivatives: $\dot{\phi}=F(\phi)+\operatorname{div} \nabla \phi$ where $F(\phi)$ is the nonconvex term. Neumann boundary conditions are universally used for $\phi$; from (10.5), setting $t=0, \epsilon_{S}=0$ to recover the standard phase-field models, we can recognize that Neumann boundary conditions are a specific choice of boundary kinetics that adapts the configuration instantaneously to always achieve zero driving force. Our use of boundary kinetics is roughly equivalent to time-dependent Dirichlet BCs, with the time-dependence being related to the fields in a complex and nonlinear fashion.

\section{Acknowledgments}

We thank ARO (W911NF-10-1-0140) for financial support. Vaibhav Agrawal also thanks the Carnegie Mellon University College of Engineering for financial support through the Bertucci Graduate Fellowship. Kaushik Dayal also thanks the Carnegie Mellon University College of Engineering for financial support through the Early Career Fellowship. This research was also supported in part by the National Science Foundation through TeraGrid resources provided by Pittsburgh Supercomputing Center. We thank Phoebus Rosakis for many useful discussions, encouragement, and pointers to the literature. We also thank the anonymous reviewers for many useful suggestions, including the suggestion to split the original single manuscript into two papers. 


\section{References}

[AV03] Rohan Abeyaratne and Srikanth Vedantam, A lattice-based model of the kinetics of twin boundary motion, Journal of the Mechanics and Physics of Solids 51 (2003), no. 9, 16751700 .

[Bha03] Kaushik Bhattacharya, Microstructure of martensite: why it forms and how it gives rise to the shape-memory effect, vol. 2, Oxford University Press, 2003.

[BJ87] JM Ball and RD James, Fine phase mixtures as minimizers of energy, Archive for Rational Mechanics and Analysis 100 (1987), no. 1, 13-52.

[BJ92] John M Ball and Richard D James, Proposed experimental tests of a theory of fine microstructure and the two-well problem, Philosophical Transactions of the Royal Society of London. Series A: Physical and Engineering Sciences 338 (1992), no. 1650, 389-450.

[BT10] IJ Beyerlein and CN Tomé, A probabilistic twin nucleation model for hcp polycrystalline metals, Proceedings of the Royal Society A: Mathematical, Physical and Engineering Science 466 (2010), no. 2121, 2517-2544.

[Cia88] Philippe G Ciarlet, Mathematical elasticity: Three dimensional elasticity, vol. 1, Studies in Mathematics and its Applications, Elsevier Science Publishers BV, Amsterdam (1988).

[CK11] John D Clayton and Jarek Knap, A phase field model of deformation twinning: nonlinear theory and numerical simulations, Physica D: Nonlinear Phenomena 240 (2011), no. 9, 841858.

[Cla10] John D Clayton, Nonlinear mechanics of crystals, vol. 177, Springer Science \& Business Media, 2010.

[GBV08] R Gröger, AG Bailey, and V Vitek, Multiscale modeling of plastic deformation of molybdenum and tungsten: I. atomistic studies of the core structure and glide of $1 / 2<111>$ screw dislocations at Ok, Acta Materialia 56 (2008), no. 19, 5401-5411.

[GRBV08] R Gröger, V Racherla, JL Bassani, and V Vitek, Multiscale modeling of plastic deformation of molybdenum and tungsten: Ii. yield criterion for single crystals based on atomistic studies of glide of 1/2<111> screw dislocations, Acta Materialia 56 (2008), no. 19, 5412-5425.

[HRL99] Thomas Y Hou, Phoebus Rosakis, and Philippe LeFloch, A level-set approach to the computation of twinning and phase-transition dynamics, Journal of Computational Physics 150 (1999), no. 2, $302-331$.

[Ka198] Surya R Kalidindi, Incorporation of deformation twinning in crystal plasticity models, Journal of the Mechanics and Physics of Solids 46 (1998), no. 2, 267-290.

[Lev14] Valery I Levitas, Phase field approach to martensitic phase transformations with large strains and interface stresses, Journal of the Mechanics and Physics of Solids (2014).

[LL07] Valery I Levitas and Dong-Wook Lee, Athermal resistance to interface motion in the phasefield theory of microstructure evolution, Physical review letters 99 (2007), no. 24, 245701. 
[LLP10] Valery I Levitas, Dong-Wook Lee, and Dean L Preston, Interface propagation and microstructure evolution in phase field models of stress-induced martensitic phase transformations, International Journal of Plasticity 26 (2010), no. 3, 395-422.

[PL13] Marcel Porta and Turab Lookman, Heterogeneity and phase transformation in materials: Energy minimization, iterative methods and geometric nonlinearity, Acta Materialia 61 (2013), no. $14,5311-5340$.

[RK97] Phoebus Rosakis and James K Knowles, Unstable kinetic relations and the dynamics of solidsolid phase transitions, Journal of the Mechanics and Physics of Solids 45 (1997), no. 11, 2055-2081.

[SB98] NK Simha and K Bhattacharya, Kinetics of phase boundaries with edges andjunctions, Journal of the Mechanics and Physics of Solids 46 (1998), no. 12, 2323-2359.

[SB99] Edge effects on the propagation of phase boundaries, Materials Science and Engineering: A 273 (1999), 241-244.

[SB00]__ Kinetics of phase boundaries with edges and junctions in a three-dimensional multiphase body, Journal of the Mechanics and Physics of Solids 48 (2000), no. 12, 2619-2641.

[WBT10] J Wang, IJ Beyerlein, and CN Tomé, An atomic and probabilistic perspective on twin nucleation in $\mathrm{mg}$, Scripta Materialia 63 (2010), no. 7, 741-746. 


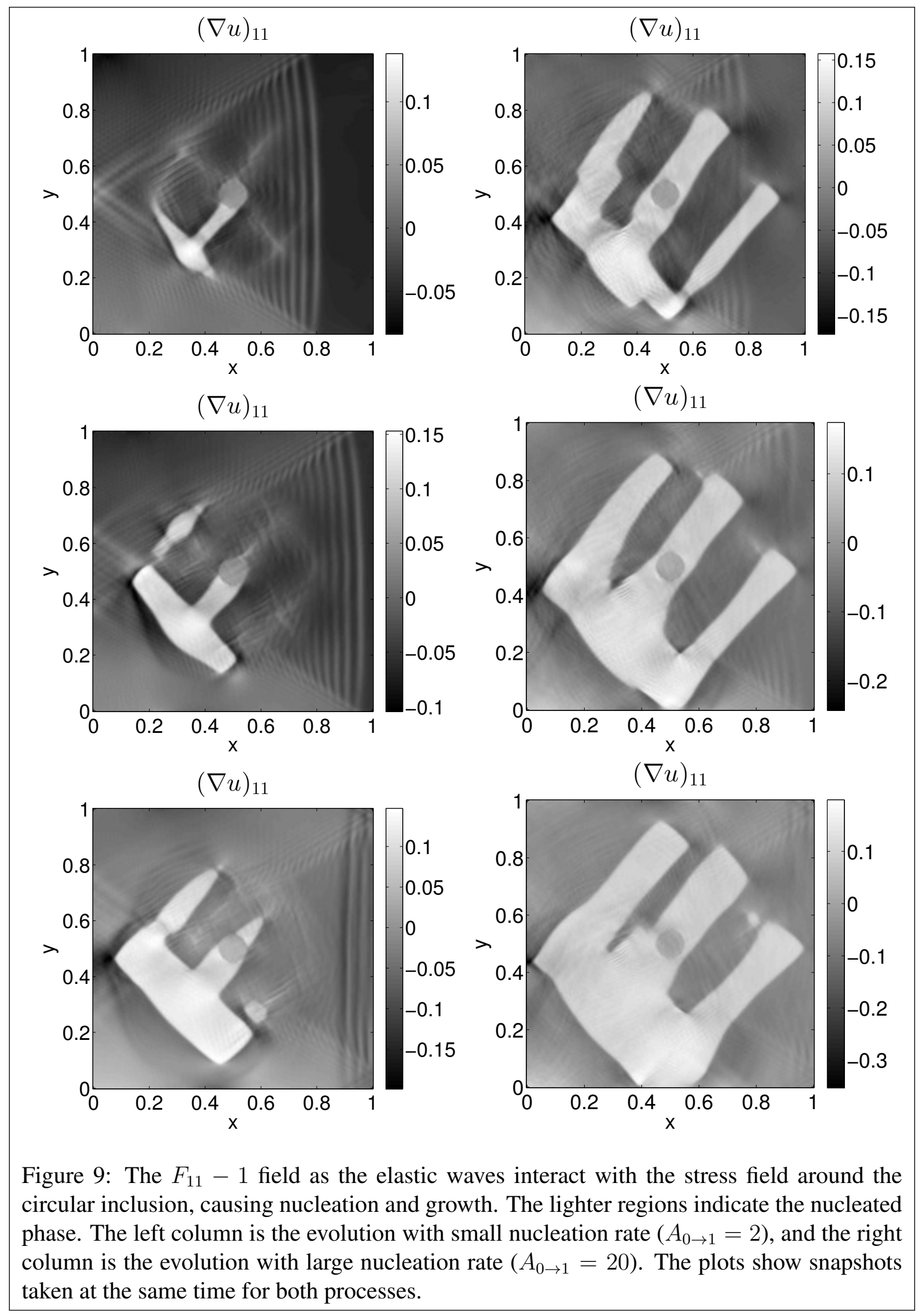




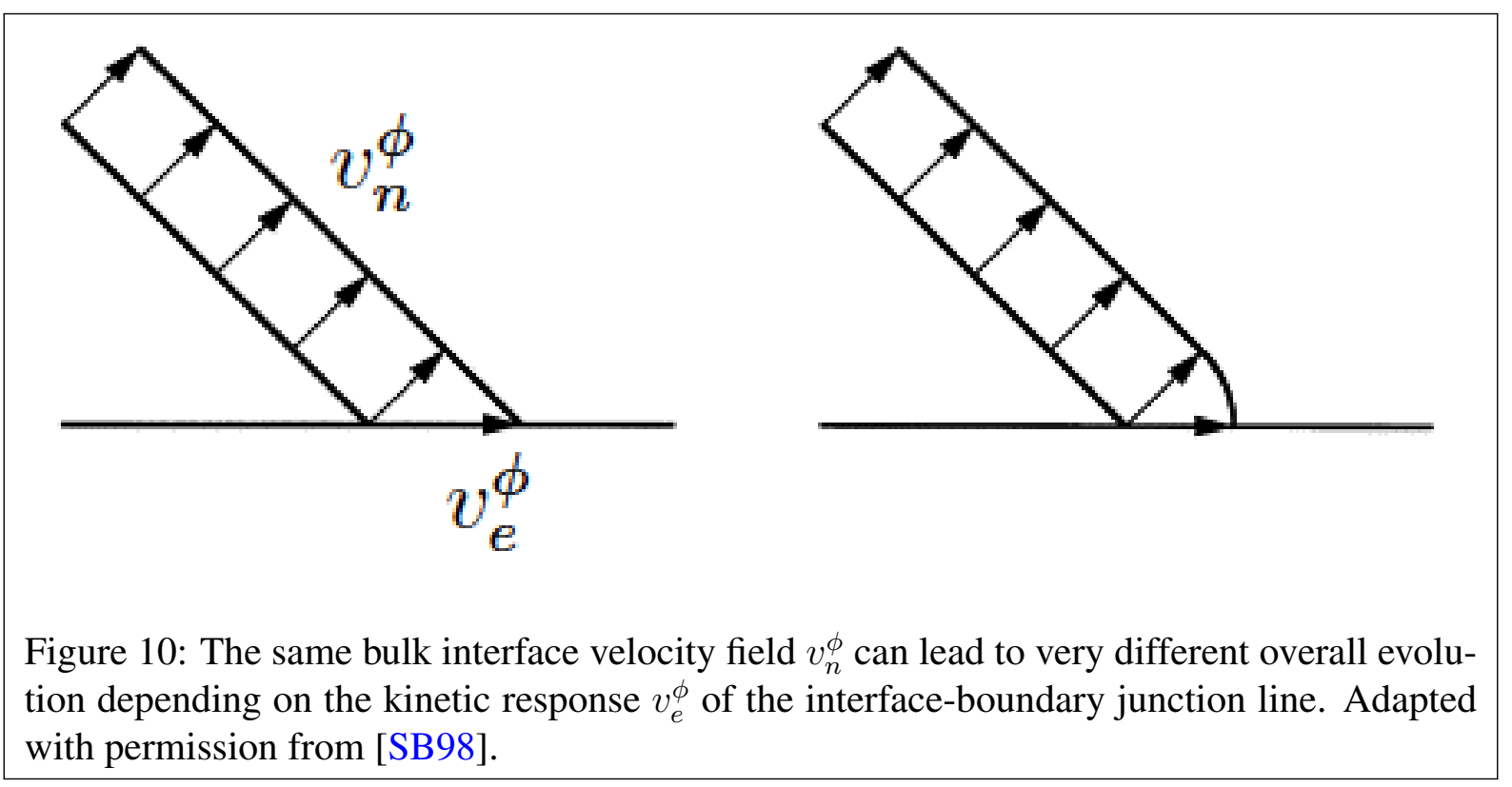




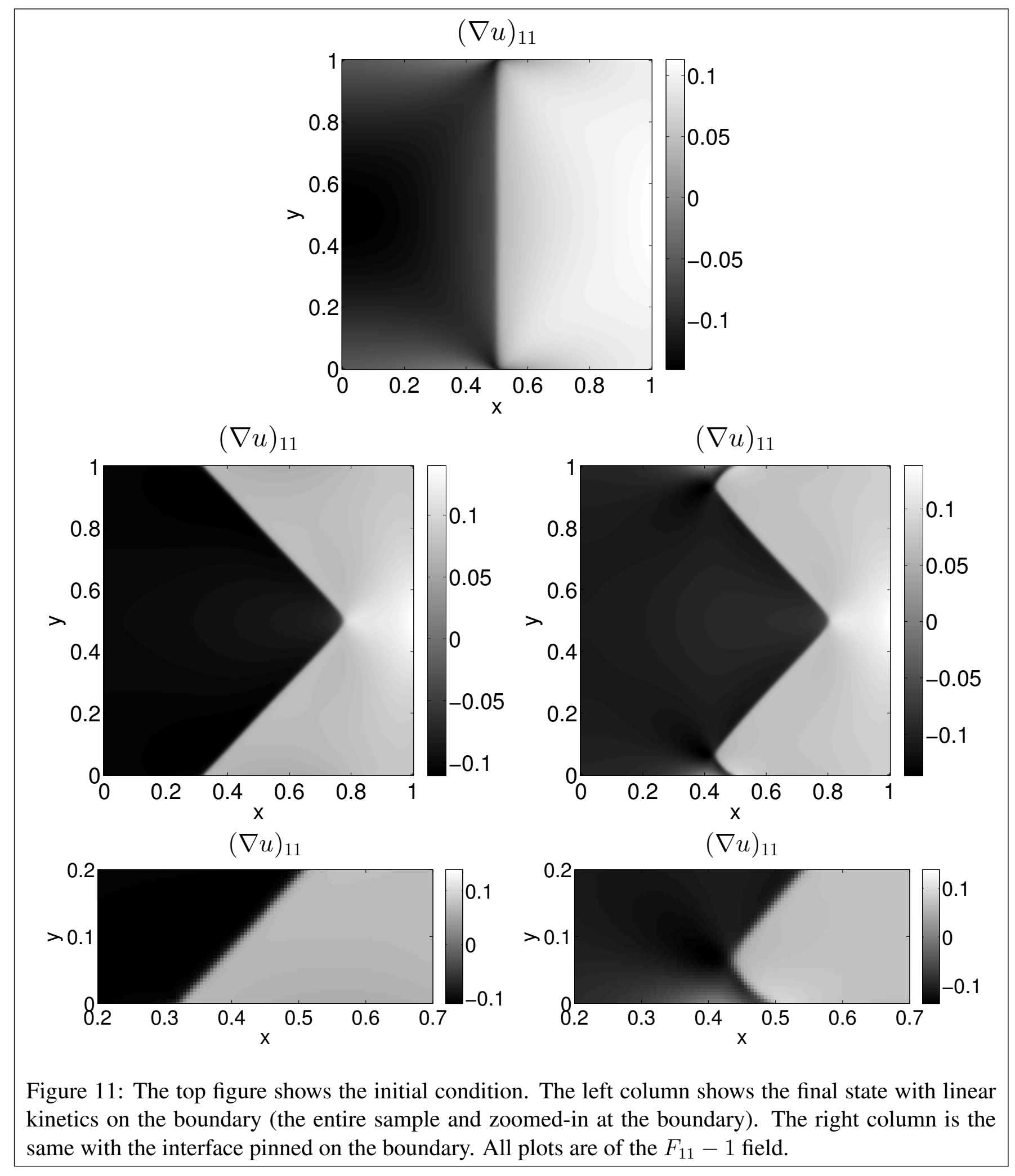



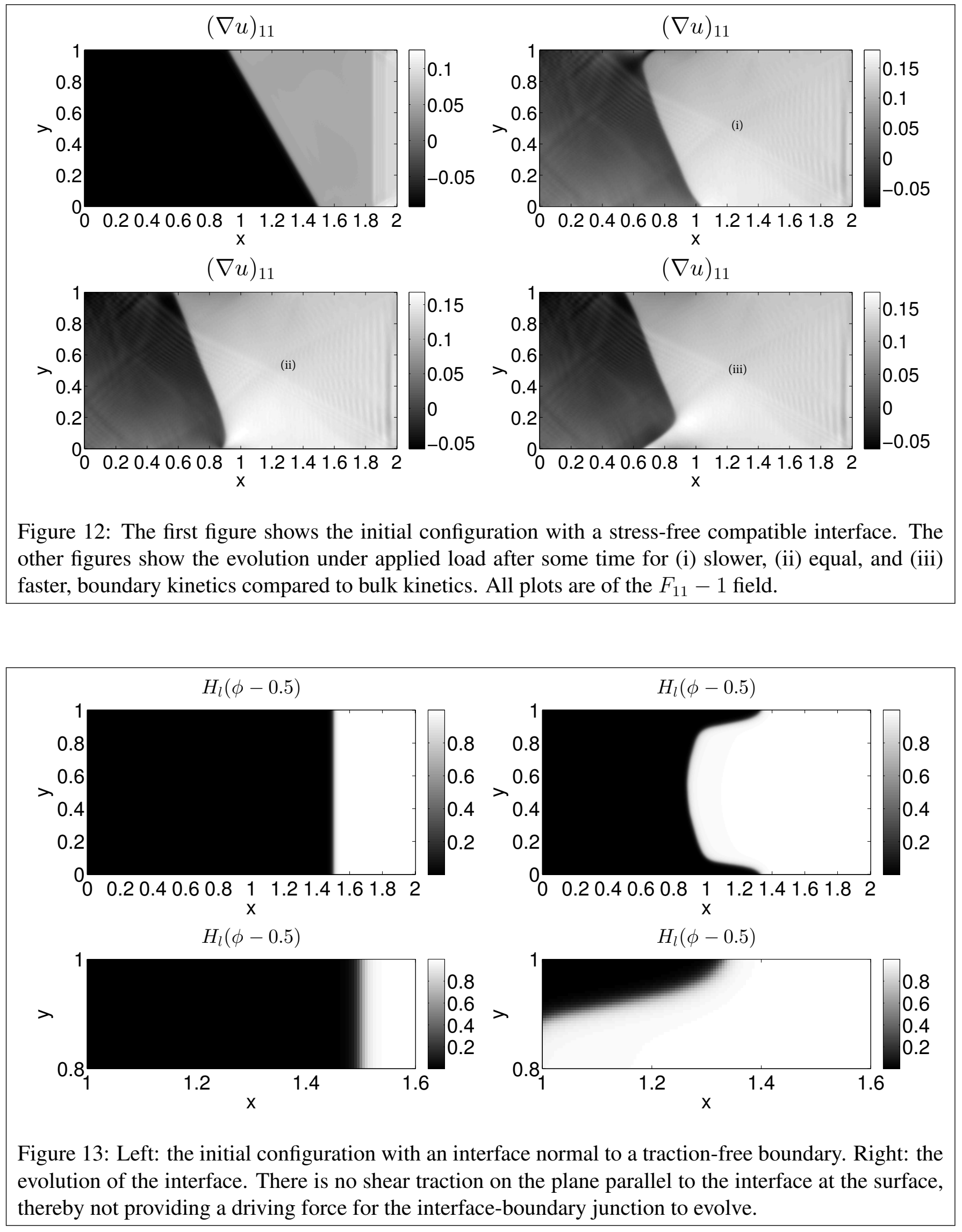